\title{
Interactive comment on "Multicriterion assessment framework of flood events simulated with the vertically mixed runoff model in semiarid catchments in the middle Yellow River" by Dayang Li et al.
}

Dayang Li et al.

libinquan@hhu.edu.cn

Received and published: 10 April 2019

Answer to RC \#1

General Comments: The paper presents a multicriterion assessment framework for flood events forecasting or warning in semi-arid regions. Four hydrologic models have been used in catchments of the middle Yellow River. The result shows that the VMM model has a better performance of flood modeling than the other three models. The 
criteria than that of in humid areas to determine whether a flood forecasting and early warning is acceptable. The topic of this study is very interesting and the idea is more or less novel. The paper is well-structured. I recommend the publication of this paper with a minor revision. Some specific comments are listed as below.

Answer: Thank you for the positive comments on our manuscript.

Specific comments: (1) The proposed framework has three parts, C1, C2 and C3. C2 is the key part of this framework, and three flow zone (low flow zone, medium flow zone, high flow zone) are divided. I think this simple framework is more important in terms of flood early warning rather than just a performance assessment. This may be real value of the framework but it is not clearly expressed in the manuscript. More explanation and discussion should be added in the paper.

Answer: Thank you for this suggestion. We have added explanations and discussions to the manuscript, especially in Section 4, Results and discussion.

(2) The initial condition is very important for a hydrologic model. In this paper, it is reasonable that the daily based model is used to calculate the initial conditions of the event-based model, but the initial condition of daily based model is not mentioned. Please add some explanations.

Answer: Thank you for this good suggestion. We have rewritten the paragraph and added more detailed information about the initial condition. The revised paragraph is as follows: "The initial condition has important effects in modeling flood events. The VMM model was run continuously from 1983 to 2009 for each catchment. Two initial values are the initial tension water storage (W0) and the initial free water storage (S0) should be determined. Both of them represent the moisture content of the soil and were assumed to be zero due to the dry conditions at 00:00:00 on January 1, 1983. Rainfall data were available only at an hourly time step over the periods of flood events, and for other periods, they were available at a daily time step. Hence, the time step of simulation was daily between flood events and hourly within flood events."

Printer-friendly version

Discussion paper 
(3) Conclusion (2): "In the four catchments, by PAWN analysis of VMM, CS, IM, and KE are the most sensitive parameters and are not affected by the choice of objective functions, whereas WM is the most sensitive parameter"make me confusing. Please use clearer and more concise expressions.

Answer: We have rewritten the sentence for clarity. "In the four catchments, the parameters confluence coefficient of surface flow (CS), impermeable area (IM), and residence time of Muskingum (KE) are the most sensitive based on an analysis by the global sensitivity method PAWN; in addition, the sensitivity ranking of the parameter WM related with the soil moisture capacity is the most affected by the objective functions."

(4) Reference/citation style needs to be revised. For example, a space is missing between Lu and et on p5, line 26; parenthesis is not right on p7, line 4, (Pianosi and Wagener,2015).

Answer: We have corrected this information.

(5) P3, line13:" Streamflow and rainfall data are from 1983 to 2009. Hourly streamflow data came from hydrological stations. Nine. ..., in this sentence, English tenses should be consistent.

Answer: Thank you for this good suggestion. We have changed this text.

(6) P3, line 26: the runoff is conceptualized as being composed of surface runoff and groundwater flow (notoriously but erroneously called "below-ground off" in the paper).

Answer: Thank you for this good suggestion. We have corrected this text.

(7) Figure 1 is requested to be further processed. The symbols of rain gauge station and hydrological station are not very clear.

Answer: We have improved the figure for clarity.

(8) Figure 3: the y-axis label may be "absolute relative error of peak flow (\%)" instead of "peak flow (\%)". The title "Figure 3: Boxplot of peak flows . . ." should be also

Printer-friendly version

Discussion paper 
checked.

Answer: We have corrected the label and checked the figure caption.

NHESSD

(9) Although I am not a native English reviewer, I find some sentences difficult to understand. The authors are encouraged to further polish up the language.

Answer: Thank you for this good suggestion. The manuscript has been polished by a professional service. All amendments are shown with tracked changes in the attached nhess-2018-402-supplement.pdf.

Please also note the supplement to this comment:

https://www.nat-hazards-earth-syst-sci-discuss.net/nhess-2018-402/nhess-2018-402AC1-supplement.pdf

Interactive comment on Nat. Hazards Earth Syst. Sci. Discuss., https://doi.org/10.5194/nhess2018-402, 2019. 